\section{ORIGINAL RESEARCH}

M.A. Rocca

G. Riccitelli

M. Rodegher

A. Ceccarelli

A. Falini

M. Falautano

A. Meani

G. Comi

M. Filippi

\title{
Functional MR Imaging Correlates of Neuropsychological Impairment in Primary-Progressive Multiple Sclerosis
}

BACKGROUND AND PURPOSE: Cognitive deficits affect $\leq 30 \%$ of patients with PPMS. We investigated the functional correlates of cognitive network dysfunction in patients with PPMS and their correlation with the extent of structural MR imaging damage.

MATERIALS AND METHODS: From 16 right-handed patients with PPMS and 17 matched controls, structural and fMRIs (during the performance of the 2-back task) were acquired. Neuropsychological tests exploring memory, attention, and frontal lobe cognitive domains were administered. T2 LL, NBV, and $\mathrm{CC}$ areas were measured.

RESULTS: Six patients with PPMS were Cl. Structural MR imaging measures did not differ between patients who were $\mathrm{Cl}$ and those who were CP. Compared with patients who were $\mathrm{Cl}$, patients who were CP had increased activations of the left caudate nucleus, PFC, and inferior parietal lobule. Compared with controls and patients who were $\mathrm{CP}$, patients who were $\mathrm{Cl}$ had increased activations of the SII, cerebellum, and insula. Compared with controls, they also had increased activations of the right precentral gyrus and a reduced recruitment of the left PFC. In patients with PPMS, a decreased composite cognitive score correlated with increased activity of the cerebellum, insula, and SII, as well as decreased PFC activity. T2 LL correlated with decreased PFC recruitment and increased SII recruitment.

CONCLUSIONS: In PPMS, an increased recruitment of cognitive-related networks might represent a functional reserve with the potential to limit the severity of cognitive impairment. The accumulation of T2 lesions and the consequent exhaustion of frontal lobe plasticity might contribute to cognitive impairment in PPMS.

ABBREVIATIONS: $\mathrm{ANOVA}=$ analysis of variance; $\mathrm{BOLD}=$ blood oxygen level-dependent; $\mathrm{CC}=$
corpus callosum; $\mathrm{Cl}=$ cognitively impaired; $\mathrm{CMA}=$ cingulate motor area; $\mathrm{CP}=$ cognitively
preserved; $\mathrm{EDSS}=$ Expanded Disability Status $\mathrm{Scale} ; \mathrm{fMRI}=$ functional $\mathrm{MR}$ imaging; $\mathrm{LL}=$ lesion
load; $\mathrm{MS}=$ multiple sclerosis; $\mathrm{NBV}=$ normalized brain volume; $\mathrm{n} . \mathrm{S}$. = not significant; PASAT =
Paced Auditory Serial Addition Test; PFC = prefrontal cortex; PPMS = primary-progressive MS;
RT = reaction times; $\mathrm{SII}=$ secondary sensorimotor cortex; $\mathrm{SPM}=$ statistical parametric mapping

$\mathbf{T}$ he mechanisms underlying disease evolution in patients with PPMS are poorly understood. Despite the accumulation of irreversible neurologic deficits, the burden and activity of lesions on conventional brain MR imaging scans are, on average, lower in PPMS than in relapsing-remitting or secondary progressive MS. ${ }^{1,2}$ The relative paucity of conventional MR imaging - detectable activity and the discrepancy between clinical and MR imaging findings observed in these patients are likely to be explained, at least partially, by the fact that the pathology of PPMS lesions consists of a predominant loss of myelin and axons with only mild inflammatory components. ${ }^{3}$ Additional factors that might contribute to such a discrepancy include the presence of diffuse tissue damage at a microscopic

Received December 2, 2009; accepted after revision January 11, 2010.

From the Neuroimaging Research Unit (M.A.R., G.R., A.C., A.M., M.Filippi), Institute of Experimental Neurology; Department of Neurology (M.A.R., M.R., M.Filippi, G.C., M.Falautano), Centro Eccellenza Risonanza Magnetica ad Alto Campo (M.A.R., A.F., M.Filippi), and Department of Neuroradiology (A.F.), Scientific Institute and University Ospedale San Raffaele, Milan, Italy.

This work was partially supported by a grant from the Fondazione Italiana Sclerosi Multipla, 2003/R/48

Please address correspondence to Massimo Filippi, MD, Neuroimaging Research Unit, Institute of Experimental Neurology, Scientific Institute and University Ospedale San Raffaele, Via Olgettina, 60, 20132 Milan, Italy; e-mail address: filippi.massimo@hsr.it

DOI 10.3174/ajnr.A2071 level, a prevalent involvement of the cervical cord, and an impairment of the adaptive capacity of the cortex to limit the functional consequences of subcortical structural injury. ${ }^{1}$

While there has been a great effort to quantify the extent of disease burden in the brain and spinal cord of patients with PPMS, only a few fMRI studies have attempted to assess the role of brain reorganization mechanisms in this disease phenotype $^{4-6}$ This is likely the consequence of the relatively low prevalence of $\mathrm{PPMS}^{1}$ and the difficulty in recruiting patients with PPMS with a relative clinical preservation, which is a critical requirement for having interpretable fMRI results. Nevertheless, the results of the available fMRI studies seem to indicate that the inefficiency of brain adaptive mechanisms might be among the factors responsible for the unfavorable disease evolution of PPMS. ${ }^{4-6}$

All the previous studies have been focused on the behavior of the motor system, which is commonly impaired by the disease. ${ }^{2}$ However, another clinical domain that is affected in $\leq 30 \%$ of patients with PPMS is cognition. ${ }^{7}$ Previous fMRI studies of cognition in patients with MS with different disease phenotypes have shown that brain plasticity might have a role in maintaining a normal level of function. ${ }^{8-13}$ However, the fMRI correlates of cognitive network dysfunction in patients with PPMS have not been investigated yet. In this study, we 
hypothesized that an abnormal functional recruitment of the cognitive network might be related to the presence and severity of cognitive impairment in patients with PPMS. To address this issue, we performed an fMRI investigation of a verbal working memory paradigm, the 2-back task, in patients with PPMS with variable cognitive impairment. In addition, to better clarify the mechanisms underlying abnormalities of function of the cognitive network in these patients, we also evaluated the correlations of fMRI findings with MR imaging measures of brain structural damage, including T2 LL and atrophy of the whole brain and the CC. The CC was selected because it is the largest compact white matter fiber bundle of the brain, which connects cortical and subcortical regions of the 2 hemispheres, thus allowing interhemispheric transfer of auditory, sensory, and motor information, which is central for maintaining normal cognitive performances.

\section{Materials and Methods}

\section{Patients}

We studied 16 consecutive patients with definite $\operatorname{PPMS}^{2}$ ( 7 men and 9 women; mean age $=49.7$ years, range $=39-68$ years; median disease duration $=10$ years, range $=4-21$ years; median EDSS score ${ }^{14}=6.0$, range $=3.0-7.0)$ and 17 healthy volunteers with no history of neurologic dysfunction and normal findings on neurologic examination ( 8 men and 9 women; mean age $=49.9$ years, range $=26-63$ years $)$. The main inclusion criteria were the following: 1) right-handedness, ${ }^{15}$ 2) absence of clinical involvement of the right upper limb and no previous relapses involving the right upper limb (for patients), 3) native Italian speaking, 4) normal or corrected-to-normal vision, and 5) no concomitant therapy with antidepressants, psychoactive drugs, or steroids. Local ethics committee approval and written informed consent from all subjects were obtained before study initiation.

\section{Functional Assessment}

Within 48 hours from MR imaging acquisition, all individuals underwent right upper limb motor functional assessment and neuropsychological evaluation. For motor functional assessment, the maximum finger tapping frequency was used. ${ }^{16}$

We assessed the following cognitive domains, which are frequently affected by $\left.\mathrm{MS}^{17-20}: 1\right)$ attention and information processing: PASAT- $3,{ }^{21}$ the Attentive Matrices Test ${ }^{22} ; 2$ ) verbal and visual-spatial memory: the Digit Span Test, Short-Story Test, Corsi Span Test, Paired Associate Word Learning Test, recall Rey-Osterrieth Complex Figure Test ${ }^{23}$; 3) abstract reasoning: the Raven Test ${ }^{24}$; 4) executive skills: the Token Test ${ }^{22}$; 5) linguistic abilities and lexical access: the phonemic and semantic verbal fluency test; and 6) spatial cognition: the copy Rey-Osterrieth Complex Figure Test. ${ }^{23}$ The individual test scores ranged from 0 to 4 , of which grade 4 means a normal performance. Patients with score 0 in at least 3 tests were considered as CI. ${ }^{7,17,25,26}$ For each patient, a cognitive impairment index was also computed as previously described. ${ }^{7}$ Briefly, the cognitive impairment index ${ }^{7}$ is a continuous variable obtained by a grading system applied to each patient's score on every cognitive test, dependent on the number of SDs below the mean normative value. These grades are then summed up across all variables to give an overall measure of cognitive dysfunction for each patient (composite cognitive score).

\section{Experimental Design}

The cognitive paradigm used in this study was the verbal $n$-back task, administered by using a block design. ${ }^{27}$ This task requires the subject to view a series of sequentially presented items and to decide whether the current item is identical to the one seen $n$ items back. We used the value of $n=2$ so that subjects were required to monitor the identity of the present item and that of the item seen 2 screens back. This choice was because we wished to test both patients who were $\mathrm{CP}$ and those who were CI, and this latter group of patients could not perform a parametric $n$-back paradigm with increasing working memory load between different conditions. Such an approach has been previously applied for the assessment of CI patients with MS. ${ }^{12}$ The verbal 2-back task was programmed with Presentation software (www.neurobs.com). Individual consonants were visually presented for $1.5 \mathrm{sec}-$ onds (followed by a black screen for 0.5 second). During each block, targets occurred with a $33 \%$ frequency in random location. ${ }^{27}$ Subjects were trained to press a button on a response box with their right hands. Accuracy of response and RT were recorded. During the resting condition, subjects had to fixate on a stationary cross in the center of the screen. All subjects underwent a training session outside the scanner before MR imaging was performed.

\section{fMRI Acquisition}

Brain MR imaging scans were obtained by using a 3T Intera scanner (Philips Healthcare, Best, the Netherlands). fMRI scans were acquired by using a $\mathrm{T}^{\star}$-weighted single-shot echo-planar imaging sequence $(\mathrm{TR}=$ 2.0 seconds, $\mathrm{TE}=30 \mathrm{~ms}$, flip angle $=85^{\circ}$, matrix size $=128 \times 128$, FOV $=240 \mathrm{~mm}^{2}$ ). During each functional scanning run, 200 sets of 30 axial sections, parallel to the anterior/posterior commissure plane, with a thickness of $4 \mathrm{~mm}$ covering the whole brain, were acquired. Each experiment consisted of 8 cycles of rest alternated with 8 cycles of task.

\section{Structural MR Imaging Acquisition}

On the same occasion, we also obtained the following images of the brain: 1$)$ a dual-echo turbo spin-echo sequence $(\mathrm{TR}=3500 \mathrm{~ms}$, $\mathrm{TE}=$ $24 / 120 \mathrm{~ms}$; echo-train length $=5$; flip angle $=150^{\circ}, 44$ contiguous 3 -mm-thick axial sections with a matrix size $=256 \times 256$ and FOV $=$ $240 \times 240 \mathrm{~mm}^{2}$ ); and 2) a 3D T1-weighted fast-field echo sequence $\left(\mathrm{TR}=25 \mathrm{~ms}, \mathrm{TE}=4.6 \mathrm{~ms}\right.$, flip angle $=30^{\circ}, 220$ contiguous, axial sections with a voxel size $=0.89 \times 0.89 \times 1 \mathrm{~mm}$, matrix size $=256 \times$ 256 , and $\mathrm{FOV}=230 \times 230 \mathrm{~mm}^{2}$ ).

\section{fMRI Analysis}

fMRI data were analyzed by using the SPM2 software (Wellcome Department of Imaging Neuroscience, London, United Kingdom) ${ }^{28}$ Before statistical analysis, all images were realigned with the first one to correct for subject motion, spatially normalized into the standard space of the SPM, and smoothed with a 10-mm 3D Gaussian filter.

\section{Structural MR Imaging Postprocessing}

T2 LL was measured by using a local thresholding segmentation technique (Jim 4.0, Xinapse System, Leicester, United Kingdom), which was applied to contour lesions on proton-attenuation-weighted images. T2-weighted scans were always used to increase confidence in lesion identification. For each subject, the area of the CC was also measured on the midsagittal section of the 3D T1-weighted images by using the same technique. On 3D T1-weighted images, NBV was measured by using the cross-sectional version of the fully automated Structural Imaging Evaluation of Normalized Atrophy software (http://www.fmrib.ox.ac.uk/fsl/siena/index.htm). ${ }^{29}$ 


\begin{tabular}{|c|c|c|c|c|c|}
\hline \multicolumn{6}{|c|}{ Structural MR imaging-derived measures from healthy volunteers and patients with PPMS } \\
\hline & $\begin{array}{l}\text { Healthy } \\
\text { Volunteers }\end{array}$ & PPMS & $P^{a}$ & CI PPMS & CP PPMS \\
\hline Mean T2 LL (mL) & $0.98 \pm 2.3$ & $8.7 \pm 10.7$ & $<.0001$ & $10.5 \pm 16.0$ & $7.4 \pm 6.1$ \\
\hline Mean NBV (mL) & $1602.1 \pm 100.3$ & $1528.3 \pm 91.3$ & .05 & $1558.7 \pm 67.1$ & $1505.5 \pm 104.3$ \\
\hline Mean CC area $\left(\mathrm{mm}^{2}\right)$ & $539.3 \pm 50.5$ & $508.0 \pm 73.7$ & n.s. & $505.5 \pm 62.4$ & $511.3 \pm 93.1$ \\
\hline
\end{tabular}

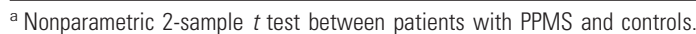

\section{Statistical Analysis}

To identify areas of brain activation during the verbal working memory task, we modeled responses for each condition on a pixel-by-pixel basis, by using the general linear model and the theory of Gaussian fields. ${ }^{28}$ In each subject, a first-level design matrix was built, in which motion parameters were used as regressors. Then, specific effects were tested by applying appropriate linear contrasts. For each subject, we defined the following contrasts: 2-back versus the resting condition and vice versa. Significant hemodynamic changes for each contrast were assessed. Then, by using an ANOVA model, a second-level random-effect analysis was performed to assess comparisons between groups. ${ }^{30}$ Intragroup activations were evaluated by using a 1-sample $t$ test. We report activations below a threshold of $P<.05$ corrected for multiple comparisons for the whole brain at a voxel level (family-wise error). A nonparametric 2 -sample $t$ test was used to compare clinical, behavioral, and structural MR imaging-derived metrics between controls and patients with PPMS and between patient subgroups. A linear regression analysis was used to assess, in patients with PPMS, the correlations between fMRI activations and neuropsychological and structural MR imaging variables $(P<.05$, corrected for multiple comparisons).

\section{Results}

\section{Functional Assessment}

No difference was found in right upper limb functional performance between patients with PPMS and controls (finger tapping rate: mean $=3.9 \pm 1.3 \mathrm{~Hz}$ for controls; mean $=3.4 \pm$ 1.2 $\mathrm{Hz}$ for patients). In the whole sample of patients with PPMS, the median value of the composite cognitive score was 31 (range $=16-36)($ composite cognitive score: median $=23$, range $=16-27$ in patients with PPMS who were CI; and median $=34$, range $=26-36$ in patients with PPMS who were $\mathrm{CP}$ ), while in healthy controls it was 42 (range $=37-46)$. Ten (62\%) patients had abnormal performance in the recall ReyOsterrieth Complex Figure Test; 9 (60\%), in the copy ReyOsterrieth Complex Figure test; 5 (31\%), in the PASAT; 4 (25\%), in Corsi Span Test; and 3 (19\%), in the Short-Story Test. The domain of visuospatial elaboration had the highest number of patients with at least 1 abnormal test result (43\%). Memory was affected in $40 \%$, and attention in $20 \%$ of the patients. None of the patients showed abnormal performance in tests exploring verbal fluency and abstract reasoning. Six patients with PPMS (37\%) had an abnormal performance in $\geq 3$ tests, thus fulfilling predefined criteria for cognitive impairment. Age, sex, disease duration, and EDSS score did not differ between patients who were CI and patients with PPMS who were CP.

\section{Structural MR Imaging}

In the Table, structural MR imaging measures from controls and patients with PPMS are reported. T2 LL was significantly higher and NBV was lower in patients with PPMS in comparison with controls. No differences were found between patients with PPMS who were CI and those who were CP.

\section{fMRI}

The analysis of behavioral data acquired during the scanning session showed no differences between patients and controls in terms of the number of errors (mean number of errors: $12.0 \pm 11.7$ in patients versus $9.5 \pm 9.5$ in controls, $P=.21$ ) and RT (mean RT during 2-back trials: $0.77 \pm 0.13$ seconds in patients versus $0.81 \pm 0.17$ in controls, $P=.39$ ). No difference was found between patients who were $\mathrm{CI}$ and those who were CP (mean number of errors: $8.8 \pm 5.2$ in patients who were $\mathrm{CI}$ versus $14.3 \pm 14.4$ in patients who were $\mathrm{CP}, P=.45$; mean $\mathrm{RT}$ during 2-back trials: $0.82 \pm 0.16$ seconds in patients who were $\mathrm{CI}$ versus $0.81 \pm 0.19$ in patients who were $\mathrm{CP}, P=.95$ ).

Areas showing BOLD-related changes at the within-group random-effects analysis during the 2-back task in controls and patients with PPMS are shown in Fig 1. Both groups had activations of several areas, which were mainly located in the frontal and parietal lobes, and included the primary and secondary sensorimotor cortices, the supplementary motor area, the CMA, the dorsolateral PFC, the insula, the basal ganglia (not shown), the cerebellum, and several other areas in the temporal and occipital lobes, bilaterally.

Compared with controls, patients with PPMS had increased activations in the cerebellum bilaterally (SPM coordinates: $-24,-50,-30$; and $18,-78,40)$, the right SII (SPM coordinates: $44,-36,16$ ), the right precentral gyrus (SPM coordinates: $56,2,32$; and $40,-18,38$ ), the right insula (SPM coordinates: $34,-2,2)$, and the left CMA (SPM coordinates: $-6,0,42)($ Fig 2$)$. No area was more active in controls versus patients.

Compared with controls, patients who were CI had more significant activations of the right precentral gyrus (SPM coordinates: 54, 4, 34), the right SII (SPM coordinates: 50, - 34, 24 ), the right insula (SPM coordinates: $34,-2,2$ ), and the cerebellum, bilaterally (SPM coordinates: 16, $-50,-26$; and $-24,-50,-30$ ), as well as reduced activations (areas more active in controls versus patients who were $\mathrm{CI}$ ) of the left PFC (SPM coordinates: $-28,52,14 ;-24,54,-10$; and $-46,32$, 14). Compared with controls, patients who were CP had more significant activations of the left PFC (SPM coordinates: -26 , $52,-8$; and $-42,8,20)$. No area was more active in controls versus patients who were CP.

Compared with patients who were CI, patients who were $\mathrm{CP}$ had more significant activations of the head of the left caudate nucleus (SPM coordinates: $-12,14,6$ ), the left PFC (SPM coordinates: $-26,54,-6$; and $-42,12,18$ ), and the left inferior parietal lobule (Brodmann area 40) (SPM coordinates: $-50,-50,50)$ (Fig 3). Conversely, compared with pa- 


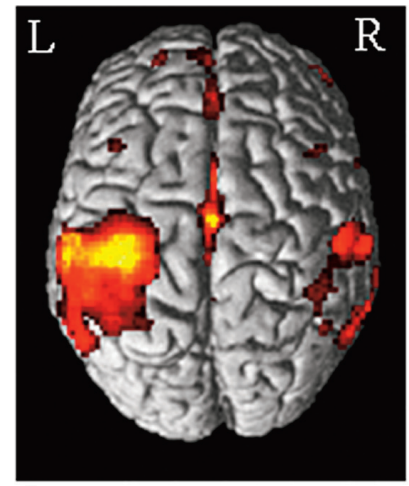

A

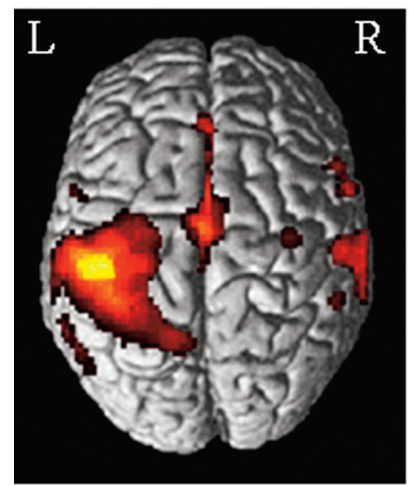

D

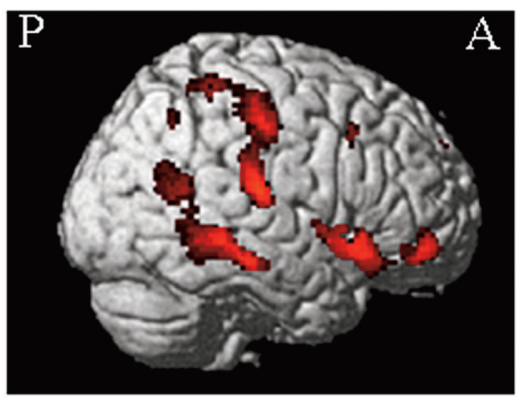

B

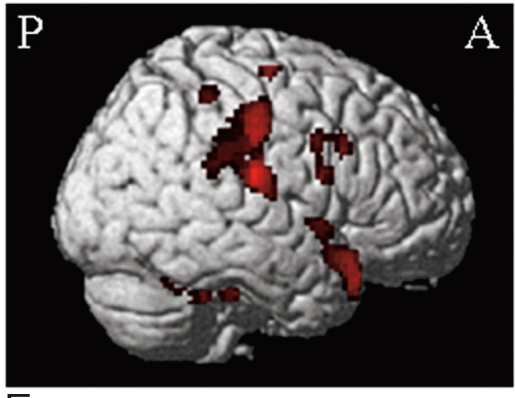

E

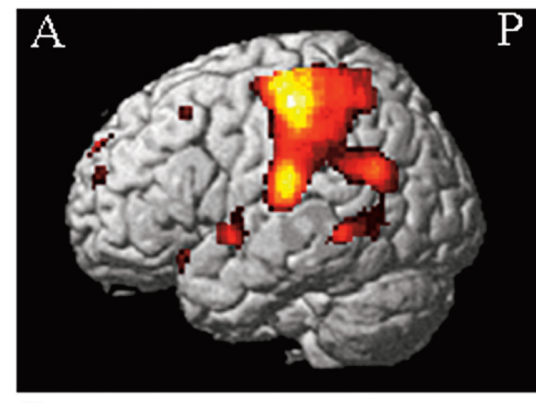

C
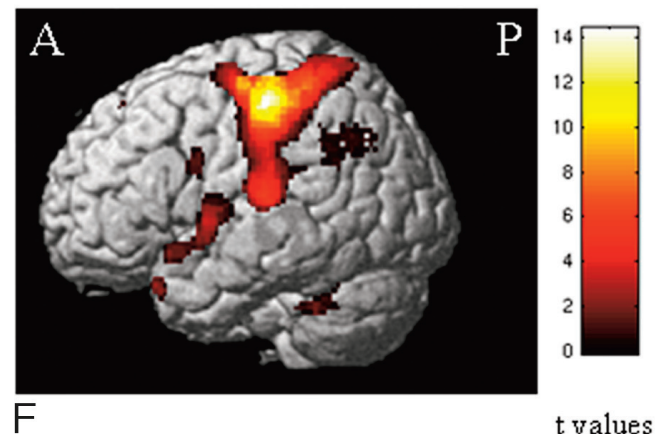

Fig 1. Areas showing BOLD changes in the within-group random-effects analysis (1-sample $t$ test, $P<.001$, uncorrected for display purposes) during the 2-back task in healthy controls $(A-C)$ and patients with PPMS $(D-F$. Note that the color-encoded activations have been superimposed on a rendered brain and normalized into the standard SPM space (neurologic convention).

tients who were CP, those with CI had more significant activations of the SII bilaterally (SPM coordinates: 50, -34, 24; and $-62,-26,24)$, the cerebellum bilaterally (SPM coordinates: $14,-50,-24$; and $-26,-44,-32$; and $-14,-54$, -24 ), and the right insula (SPM coordinates: 40, 4, 2) (Fig 3 ).

\section{Correlations of fMRI Findings with Neuropsychological and Structural MR Imaging Measures}

In the entire sample of patients with PPMS, significant correlations $(P<.05)$ were found between the following:

1) Decrease of the composite cognitive score and increased activity of the left cerebellum $(r=-0.87)$ and the right SII $(r=-0.90)$.

2) Increase of T2 LL and decreased recruitment of the left PFC $(r=-0.91)$ and increased recruitment of the right SII $(r=$ $0.80)$.

No correlation was found between fMRI and atrophy measures, or between the composite cognitive score and structural MR imaging-derived measures.

\section{Discussion}

This is the first fMRI study performed to interrogate the mechanisms associated with the presence of cognitive deficits in patients with PPMS. To try to achieve a comprehensive picture of brain reorganization at different stages of cognitive impairment, we studied patients with different degrees of cognitive deficits. One of the main issues when using a cognitive para- digm to study patients who are CI is to obtain interpretable data, suitable for a comparison with results derived from CP subjects. To this aim, we carefully selected right-handed subjects without overt right-hand motor impairment and used a simplified version of the $n$-back task, which has been demonstrated to activate the working memory network. ${ }^{12,31}$ This might be the reason that we did not find a significant behavioral difference during task performance between patients who were CP and those who were CI.

Consistent with previous reports of the 2-back effect on fMRI activations in healthy people, ${ }^{31-33}$ all subjects showed activations of several areas located in the frontal and parietal lobes (including the primary sensorimotor cortex, the supplementary motor area, the CMA, the inferior parietal lobule, and the SII), the temporo-occipital lobes, the insula, the basal ganglia, and the cerebellum. All of these regions contribute to different aspects of working memory processing, and most of them have been demonstrated to have a load-depending activity during working memory tasks. ${ }^{27}$ Activation of the sensorimotor network can be attributed to the motor response required during the task. The cingulum is supposed to have a crucial role in attention and response selection. ${ }^{34,35}$ Sensory association areas in the parietal and temporal lobes contribute to maintaining stimulus representation in memory when the original stimulus is withdrawn from vision. ${ }^{36}$ Finally, the cerebellum is known to be involved in high-level cognitive functions and conflict adaptation. ${ }^{37}$

The comparison of the brain patterns of activation between 

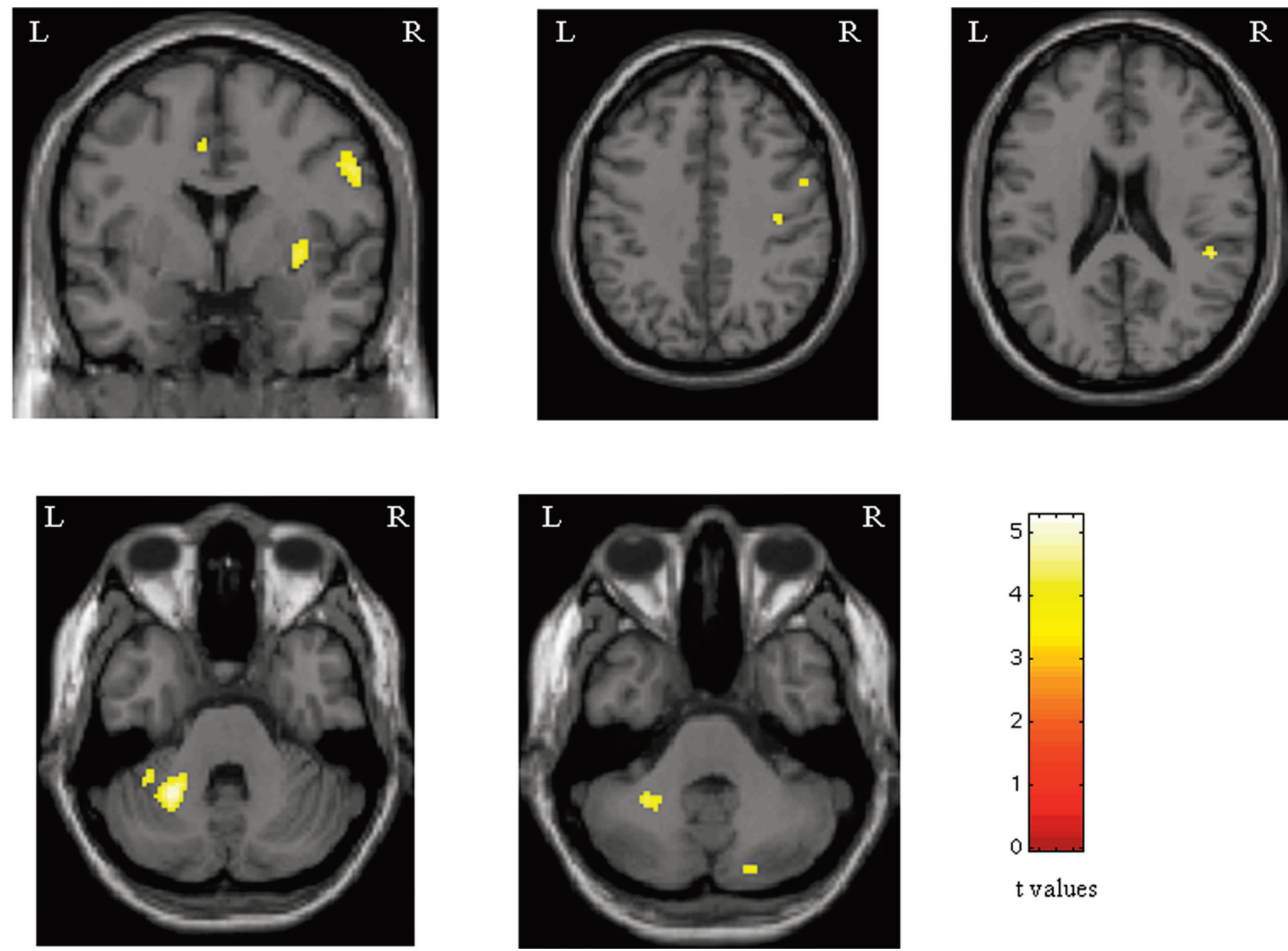

Fig 2. Areas showing increased activations in patients with PPMS in comparison with healthy controls during the analysis of the 2-back task (random-effect-analysis, ANOVA, $P<.05$ corrected for multiple comparisons). Note that the color-encoded activations have been superimposed on a rendered brain and normalized into the standard SPM space (neurologic convention). See text for further details.

patients with PPMS and controls demonstrated, in the former group, an increased recruitment of several areas, which have a critical role in tasks involving working memory, such as the cerebellum, the SII, the CMA, the insula, and the precentral gyrus. Several fMRI studies of cognitive network function in patients with MS with different disease phenotypes have demonstrated that the increased activation of specialized areas of a given network, as well as their bilateral recruitment, are among the adaptive mechanisms responsible for the preservation of function. ${ }^{8-10,38}$ Our results support the notion that these mechanisms are also likely to have a role in patients with PPMS.

To disentangle the factors potentially related to cognitive impairment in patients with PPMS, we compared the taskrelated activation between patients with cognitive impairment versus those without cognitive impairment and healthy controls. This analysis revealed a decreased activation of the PFC in patients who were CI. In addition, patients who were $\mathrm{CI}$ had an increased recruitment of the cerebellum, the insula, and the SII. The PFC is an important component of the neural substrate for working memory. This structure has been involved in a variety of working memory tasks, in conjunction with more posterior regions, such as areas located in the parietal lobes. ${ }^{27,39}$ Previous studies of the working memory network in patients with relapsing-remitting MS and intact cognitive per- formance have shown a reduced ability to optimize PFC recruitment in patients with MS with increasing task difficulty. ${ }^{40}$ In this perspective, our results suggest that the exhaustion of the functional properties of this region is likely to play a critical role in the development of cognitive deficits in MS. Our findings also indicate that posterior regions might, at least to some extent, take over the function of the PFC during the performance of a working memory task, thus contributing to the maintenance of a normal level of function.

The concept that changes in the interactions between different brain areas may alter the cognitive network efficiency in MS agrees with studies of functional and effective connectivity in relapsing-remitting $\mathrm{MS}^{8,40}$ and benign $\mathrm{MS}^{20}{ }^{20}$ which have shown either decreased or increased connectivity. These authors ${ }^{8,40,41}$ interpreted such changes as a compensatory adaptation of the studied networks to the underlying disease-related structural damage. This might not be the case in patients with PPMS, in whom the measured fMRI changes do not necessarily reflect an adaptive mechanism, as suggested by the correlation analysis of functional data with neuropsychological assessment. Remarkably, this analysis, which was performed on the entire group of patients with PPMS, revealed a direct correlation between the degree of cognitive impairment, measured by using the composite cognitive score, and the reduced activation of the PFC, as well as an inverse linear 


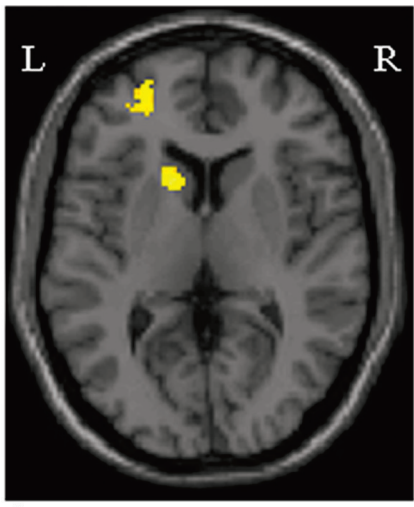

$\bar{A}$

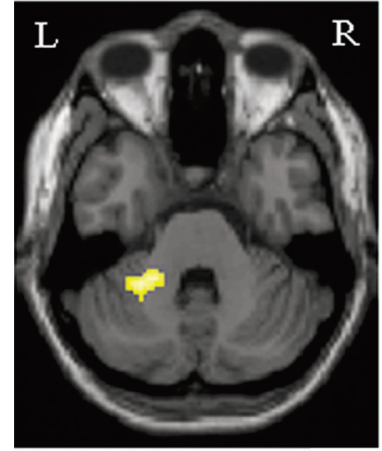

$\mathrm{D}$

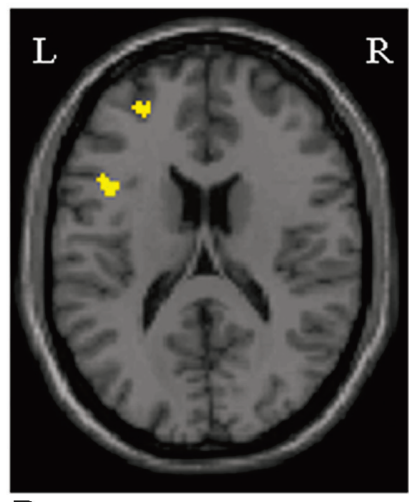

$\bar{B}$

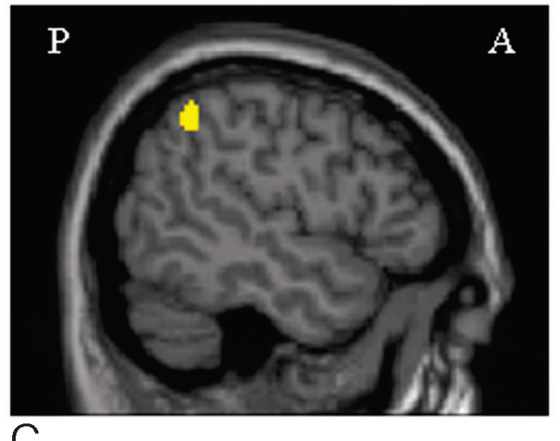

C

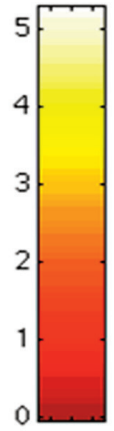

$t$ values

Fig 3. Areas of increased activations in patients with PPMS without cognitive impairment vs. those with cognitive impairment $(A-C)$, and areas of increased activations in patients with PPMS with cognitive impairment vs. those without $(D-G)$ during the analysis of the 2-back task (random-effects analysis, ANOVA, $P<.05$ corrected for multiple comparisons). Note that the color-encoded activations have been superimposed on a rendered brain and normalized into the standard SPM space (neurologic convention). See text for further details.

relationship between the composite cognitive score and increased fMRI recruitment of the cerebellum, insula, and SII, suggesting that such an over-recruitment might reflect a maladaptive response possibly related to tissue injury. This notion is in line with previous results in patients at risk of advancing to definite MS, ${ }^{16}$ in whom the increased recruitment of regions of the motor network has been shown to predict the subsequent evolution to definite disease. Our hypothesis of a "maladaptive" response in patients with PPMS is also in line with the results from previous studies of the motor system in these patients, ${ }^{4-6}$ which have suggested a lack or a precocious exhaustion of the "classic" adaptive mechanism in this disease phenotype.

To improve our understanding of the structural substrates of functional changes in patients with PPMS, we assessed the correlation between fMRI changes and MR imaging metrics of structural damage, including T2 LL, atrophy of the whole brain, and atrophy of the CC. Only the extent of T2 lesion burden was correlated with loss of function in the PFC and increased recruitment of the SII, thus supporting the hypothesis that a progressive dysfunction of the frontal lobe, due to the accumulation of brain lesions, might be among the factors responsible for the development of cognitive deficits in these patients. Indeed, MS lesions are typically located in the periventricular regions, and such a distribution is likely to cause a disruption of white matter fiber bundles connecting frontal and parietal lobes, which in turn may result in a reduced functional interaction.
Clearly, this study has limitations. First, it was cross-sectional, and longitudinal studies are needed to strengthen the validity of our findings. Second, due to our stringent inclusion criteria, the sample size was relatively small. Third, we did not quantify damage in the normal-appearing brain tissue, which is also likely to contribute to the observed cognitive and fMRI abnormalities. Fourth, we did not acquire fast fluid-attenuated inversion recovery sequences, which might result in a different harvest of focal WM lesions than dual-echo scans. Finally, due to the length of the MR imaging acquisition protocol and patients' disabilities, we limited our analysis to a single fMRI paradigm, which, however, was focused on a cognitive domain typically affected by the disease.

\section{Conclusions}

In this study, we hypothesized that the recruitment of the cognitive network in patients with PPMS might vary according to the severity of their cognitive impairment and that such an abnormal recruitment might be related to the extent of structural brain damage. We found that the regions engaged during the performance of a cognitive task differed between patients who were $\mathrm{CP}$ and those who were $\mathrm{CI}$ with PPMS, with a prominent recruitment of areas in the frontal lobes in patients who were $\mathrm{CP}$ and parietal and cerebellar regions in patients who were CI. Such fMRI findings were related to T2 lesion burden, suggesting that accumulation of $\mathrm{T} 2$ lesions and the consequent exhaustion of frontal lobe plasticity might contribute to cognitive impairment in PPMS. 


\section{References}

1. Miller DH, Leary SM. Primary-progressive multiple sclerosis. Lancet Neurol 2007;6:903-12

2. Thompson AJ, Montalban X, Barkhof F, et al. Diagnostic criteria for primary progressive multiple sclerosis: a position paper. Ann Neurol 2000;47:831-35

3. Revesz T, Kidd D, Thompson AJ, et al. A comparison of the pathology of primary and secondary progressive multiple sclerosis. Brain 1994;117(pt 4):759-65

4. Ciccarelli O, Toosy AT, Marsden JF, et al. Functional response to active and passive ankle movements with clinical correlations in patients with primary progressive multiple sclerosis. J Neurol 2006;253:882-91

5. Filippi M, Rocca MA, Falini A, et al. Correlations between structural CNS damage and functional MRI changes in primary progressive MS. Neuroimage 2002;15:537-46

6. Rocca MA, Matthews PM, Caputo D, et al. Evidence for widespread movement-associated functional MRI changes in patients with PPMS. Neurology 2002;58:866-72

7. Camp SJ, Stevenson VL, Thompson AJ, et al. Cognitive function in primary progressive and transitional progressive multiple sclerosis: a controlled study with MRI correlates. Brain 1999;122(pt 7):1341-48

8. Audoin B, Ibarrola D, Ranjeva JP, et al. Compensatory cortical activation observed by fMRI during a cognitive task at the earliest stage of MS. Hum Brain Mapp 2003;20:51-58

9. Hillary FG, Chiaravalloti ND, Ricker JH, et al. An investigation of working memory rehearsal in multiple sclerosis using fMRI. J Clin Exp Neuropsychol 2003;25:965-78

10. Mainero C, Caramia F, Pozzilli C, et al. fMRI evidence of brain reorganization during attention and memory tasks in multiple sclerosis. Neuroimage 2004;21:858-67

11. Mainero $C$, Pantano $P$, Caramia $F$, et al. Brain reorganization during attention and memory tasks in multiple sclerosis: insights from functional MRI studies. J Neurol Sci 2006;245:93-98

12. Penner IK, Rausch M, Kappos L, et al. Analysis of impairment related functional architecture in MS patients during performance of different attention tasks. J Neurol 2003;250:461-72

13. Rocca MA, Filippi M. Functional MRI in multiple sclerosis. J Neuroimaging 2007;17(suppl 1):36S-41S

14. Kurtzke JF. Rating neurologic impairment in multiple sclerosis: an expanded disability status scale (EDSS). Neurology 1983;33:1444-52

15. Oldfield RC. The assessment and analysis of handedness: the Edinburgh inventory. Neuropsychologia 1971;9:97-113

16. Rocca MA, Mezzapesa DM, Ghezzi A, et al. A widespread pattern of cortical activations in patients at presentation with clinically isolated symptoms is associated with evolution to definite multiple sclerosis. AJNR Am J Neuroradiol 2005;26:1136-39

17. Comi G, Filippi M, Martinelli V, et al. Brain MRI correlates of cognitive impairment in primary and secondary progressive multiple sclerosis. J Neurol Sci 1995;132:222-27

18. Rovaris M, Riccitelli G, Judica E, et al. Cognitive impairment and structural brain damage in benign multiple sclerosis. Neurology 2008;71:1521-26

19. Mesaros S, Rocca MA, Riccitelli G, et al. Corpus callosum damage and cognitive dysfunction in benign MS. Hum Brain Mapp 2009;30:2656-66
20. Rocca MA, Valsasina P, Ceccarelli A, et al. Structural and functional MRI correlates of Stroop control in benign MS. Hum Brain Mapp 2009;30:276-90

21. Gronwall DM. Paced auditory serial-addition task: a measure of recovery from concussion. Percept Mot Skills 1977;44:367-73

22. Spinnler H, Tognomi G. Standardizzazione e taratura italiana di test neuropsicologici. Ital J Neurol Sci 1987;6:1-20

23. Caffarra P, Vezzadini G, Dieci F, et al. Rey-Osterrieth complex figure: normative values in an Italian population sample. Neurol Sci 2002;22:443-47

24. Basso A, Capitani E, Laiacona M. Raven's coloured progressive matrices: normative values on 305 adult normal controls. Funct Neurol 1987;2:189-94

25. Amato MP, Zipoli V, Portaccio E. Multiple sclerosis-related cognitive changes: a review of cross-sectional and longitudinal studies. J Neurol Sci 2006; 245:41-46

26. Amato MP, Zipoli V, Portaccio E. Cognitive changes in multiple sclerosis. Expert Rev Neurother 2008;8:1585-96

27. Braver TS, Cohen JD, Nystrom LE, et al. A parametric study of prefronta cortex involvement in human working memory. Neuroimage 1997;5:49-62

28. Friston KJ, Holmes AP, Poline JB, et al. Analysis of fMRI time-series revisited. Neuroimage 1995;2:45-53

29. Smith SM, Zhang Y, Jenkinson M, et al. Accurate, robust, and automated longitudinal and cross-sectional brain change analysis. Neuroimage 2002; 17:479-89

30. Friston KJ, Holmes AP, Price CJ, et al. Multisubject fMRI studies and conjunction analyses. Neuroimage 1999;10:385-96

31. Owen AM, McMillan KM, Laird AR, et al. N-back working memory paradigm: a meta-analysis of normative functional neuroimaging studies. Hum Brain Mapp 2005;25:46-59

32. Courtney SM, Ungerleider LG, Keil K, et al. Object and spatial visual working memory activate separate neural systems in human cortex. Cereb Cortex 1996;6:39-49

33. Smith EE, Jonides J, Koeppe RA. Dissociating verbal and spatial working memory using PET. Cereb Cortex 1996;6:11-20

34. Corbetta M, Miezin FM, Dobmeyer S, et al. Selective and divided attention during visual discriminations of shape, color, and speed: functional anatomy by positron emission tomography. J Neurosci 1991;11:2383-402

35. Paus T, Petrides M, Evans AC, et al. Role of the human anterior cingulate cortex in the control of oculomotor, manual, and speech responses: a positron emission tomography study. J Neurophysiol 1993;70:453-69

36. Muller NG, Knight RT. The functional neuroanatomy of working memory: contributions of human brain lesion studies. Neuroscience 2006;139:51-58

37. Akshoomoff NA, Courchesne E. A new role for the cerebellum in cognitive operations. Behav Neurosci 1992;106:731-38

38. Wishart HA, Saykin AJ, McDonald BC, et al. Brain activation patterns associated with working memory in relapsing-remitting MS. Neurology 2004; 62:234-38

39. McCarthy G, Blamire AM, Puce A, et al. Functional magnetic resonance imaging of human prefrontal cortex activation during a spatial working memory task. Proc Natl Acad Sci U S A 1994;91:8690-94

40. Cader S, Cifelli A, Abu-Omar Y, et al. Reduced brain functional reserve and altered functional connectivity in patients with multiple sclerosis. Brain 2006;129:527-37

41. Rocca MA, Pagani E, Absinta M, et al. Altered functional and structural connectivities in patients with MS: a 3-T study. Neurology 2007;69:2136-45 